\title{
Invited Capstone Talk Some Industrial Experience with Teaching Formal Methods
}

Rod Chapman

Praxis High Integrity Systems

20 Manvers Street, Bath BA1 1PX, UK

rod.chapman@praxis-his.com

\begin{abstract}
This presentation will reflect on the experience of teaching formal methods in an industrial context. Our experience includes teaching formal specification using $Z$ and program verification using the SPARK language and tools. Our results are surprisingly positive - industrial engineers (from a wide range of academic backgrounds) seem capable of taking on and applying these notations and techniques. The presentation will also offer some reflection on tools and how they present formal techniques to their users.
\end{abstract}

